\title{
Model study of generalized parton distributions with helicity flip
}

\author{
Sergio Scopetta \\ Dipartimento di Fisica, Università degli Studi di Perugia, and INFN, sezione di Perugia \\ via A. Pascoli 06100 Perugia, Italy,
}

\begin{abstract}
Generalized parton distributions with helicity flip are studied in the quark sector, within a simple version of the MIT bag model, assuming an SU(6) wave function for the proton target. In the framework under scrutiny it turns out that only the generalized transversity distribution, $H_{T}^{q}$, is non vanishing. For this quantity, the forward limit is properly recovered and numerical results are found to underestimate recent lattice data for its first moment. Positivity bounds recently proposed are fulfilled by the obtained distribution. The relevance of the analysis for the planning of measurements of the quark generalized transversity is addressed.
\end{abstract}

Typeset using REVTEX 
The distribution of transverse quark spin is one of the least known features of nucleon structure. In a few years, some light should be shed on it by experiments [1]. The possibility of measuring the generalized transverse spin distribution is also under scrutiny, establishing a link between transversity and Generalized Parton Distributions (GPDs) [2]. GPDs represent one of the main topics of interest in nowadays hadronic physics [3]. At twist-two, eight GPDs occur. Four of them, helicity conserving ones, enter processes where the helicity of the parton is conserved. They are labelled $H, E, \tilde{H}, \tilde{E}$ and have been extensively studied and modelled. The other four twist-two GPDs, $H_{T}, E_{T}, \tilde{H}_{T}, \tilde{E}_{T}$, the subject of this study, are parton helicity flip ones and have been introduced in Ref. [4], although their correct classification and counting have been established later, in Ref. [5]. Being diagonal in a transversity basis, they are also called "transversity GPDs". I prefer to call them "GPDs with helicity flip", calling generalized transversity distribution the quantity $H_{T}$, the only one which survives in the forward limit, yielding the transversity density, $h_{1}$. While gluon helicity flip GPDs appear at leading twist in Deeply Virtual Compton Scattering [6], the same does not occur in the quark sector, not even in hard exclusive electroproduction of mesons [7]. Diffractive double meson production is the only process which is known to give access to the quark generalized transversity, in the Efremov-Radyushkin-Brodsky-Lepage (ERBL) region [8]. An estimate of helicity flip GPDs would help to study the feasibility of such an experiment. Recently, lattice data for their lowest moments [9] and a study of positivity bounds on them have appeared [10]. Studies of helicity flip GPDs in the transverse plane have been completed [11]. Anyway, to my knowledge, a model calculation of these quantities has not been performed yet. In here, a model estimate of quark GPDs with helicity flip is presented. The analysis is performed within the MIT bag model [12], assuming SU(6) symmetry, following the lines of Ref. [13], where helicity conserving GPDs have been calculated. Despite of well known drawbacks, such as the breaking of translational invariance, the MIT bag model has proven to be able to provide reasonable initial inputs, at a low factorization scale, for the unpolarized [14], polarized [15], transversity [16,17] and orbital angular momentum [18] distributions. The MIT bag model has been also the 
framework for the first estimate of helicity conserving GPDs [13], up to twist three [19], and it represents therefore the natural playground for the first analysis of the helicity flip ones.

The main quantities of interest are now defined. Quark helicity flip GPDs, are introduced through the relation [5]

$$
\begin{aligned}
\left.\frac{1}{2} \int \frac{d z^{-}}{2 \pi} e^{i x P^{+} z^{-}}\left\langle p^{\prime}, \lambda^{\prime}\left|\bar{\psi}_{q}\left(-\frac{1}{2} z\right) i \sigma^{+i} \psi_{q}\left(\frac{1}{2} z\right)\right| p, \lambda\right\rangle\right|_{z^{+}=0, \mathbf{z}_{\mathbf{T}}=\mathbf{0}} \\
=\frac{1}{2 P^{+}} \bar{u}\left(p^{\prime}, \lambda^{\prime}\right)\left[H_{T}^{q} i \sigma^{+i}+\tilde{H}_{T}^{q} \frac{P^{+} \Delta^{i}-\Delta^{+} P^{i}}{m^{2}}\right. \\
\left.+E_{T}^{q} \frac{\gamma^{+} \Delta^{i}-\Delta^{+} \gamma^{i}}{2 m}+\tilde{E}_{T}^{q} \frac{\gamma^{+} P^{i}-P^{+} \gamma^{i}}{m}\right] u(p, \lambda),
\end{aligned}
$$

where $p, p^{\prime}$ and $\lambda, \lambda^{\prime}$ respectively denote momenta and helicities of the nucleon and $i=1,2$ is a transverse index. Use is made of light-cone coordinates $\left(v^{ \pm}=\left(v^{0} \pm v^{3}\right) / \sqrt{2}\right.$ and $\mathbf{v}_{\mathbf{T}}=$ $\left(v^{1}, v^{2}\right)$ for any four-vector $\left.v\right)$ and of Ji's kinematical variables, $P=\left(p+p^{\prime}\right) / 2, \Delta=p^{\prime}-p, \xi=$ $\left(p^{+}-p^{++}\right) /\left(p^{+}+p^{+}\right)=-\Delta^{+} / 2 P^{+}$, and $t=\Delta^{2}$. It is convenient to use light-cone helicity states and to have the quarks on shell, so that the operators occurring in the definitions of the quark distributions have the simplest structure. With this choice the helicity flip GPDs turn out to be related to the matrix elements $A_{\lambda^{\prime} \mu^{\prime}, \lambda \mu}$, for definite parton helicities $\mu^{\prime}=+$ and $\mu=-$

$$
\begin{aligned}
A_{\lambda^{\prime}+, \lambda-}^{q} & =\left.\int \frac{d z^{-}}{2 \pi} e^{i x P^{+} z^{-}}\left\langle p^{\prime}, \lambda^{\prime}\left|\mathcal{O}_{+,-}^{q}(z)\right| p, \lambda\right\rangle\right|_{z^{+}=0, \mathbf{z}_{\mathbf{T}}=\mathbf{0}} \\
& =\int \frac{d^{2} k_{T}}{(2 \pi)^{3}}\left[\int d z^{-} d^{2} z_{T} e^{i k \cdot z}\left\langle p^{\prime}, \lambda^{\prime}\left|\mathcal{O}_{+,-}^{q}(z)\right| p, \lambda\right\rangle\right]_{z^{+}=0, k^{+}=x P^{+}}
\end{aligned}
$$

with the operator $\mathcal{O}_{+,-}^{q}(z)$, given by

$$
\mathcal{O}_{+,-}^{q}(z)=\frac{i}{4} \bar{\psi}_{q}\left(-\frac{z}{2}\right)\left(\sigma^{+1}+i \sigma^{+2}\right) \psi_{q}\left(\frac{z}{2}\right)
$$

flipping the parton helicity from $\mu=-\frac{1}{2}$ to $\mu^{\prime}=\frac{1}{2}$ [5]. In a model study of GPDs with helicity flip, the crucial calculation is therefore the evaluation of the matrix elements Eq. (2). Once these results are available, the GPDs are obtained from them and their explicit form can be found, i.e., in Ref. [10].

The procedure of Ref. [13] for estimating GPDs is adopted here, using a simple version of the MIT bag model, able to reproduce the gross features of parton distributions [14-18] 
and form factors (ff) [20], despite of its drawbacks later discussed. When evaluating GPDs it is convenient to work in the Breit frame, where $p_{\mu}=(\bar{m} ;-\vec{\Delta} / 2), p_{\mu}^{\prime}=(\bar{m} ; \vec{\Delta} / 2)$, $t=\Delta^{2}=-\vec{\Delta}^{2}=4\left(m^{2}-\bar{m}^{2}\right)$ and $\xi=-\Delta_{z} /(2 \bar{m})$. In principle, since translational invariance is violated, different results will be obtained in different frames. As in Ref [13], it is assumed here that the results are weakly frame dependent. The calculation, performed for quarks of minimum energy in the bag, requires wave functions of moving nucleons and one has to boost the rest frame wave function to a moving frame [20]. Here, following Ref. [13], a simple prescription is used to partially restore momentum conservation, taking the momentum transfer through the active quark to be $\eta \vec{\Delta}$, where $\eta$ is a parameter to be fixed by fitting the nucleon electromagnetic ff [20]. In Ref. [13], it was found that small $|t|$ data favor a value of $\eta=0.55$, while a better fit is achieved at larger $|t|$ with $\eta=0.35$.

Following this approach, I obtained the following expression for the matrix elements Eq. $(2)$ :

$$
\begin{aligned}
A_{\lambda^{\prime}+, \lambda-}^{q}(x, \xi, t) & =\sqrt{1-\xi^{2}} Z^{2}(t)\left(4 \pi N^{2} R^{6}\right) \frac{\bar{m}}{1-C_{1} \Delta_{z}^{2} / t} \int \frac{d k_{\perp} d \varphi}{(2 \pi)^{3}} k_{\perp} \\
& \times\left\{C t_{0}(k) t_{0}\left(k^{\prime}\right)+C \frac{k_{z}}{k} t_{1}(k) t_{0}\left(k^{\prime}\right)\right. \\
& +\left[C+2 \frac{\Delta_{x} \widetilde{\Delta}_{x}}{|\vec{\Delta}| k_{z}^{\prime}}\left(\cosh \frac{\omega}{2} \sinh \frac{\omega}{2}-\frac{\Delta_{z}}{|\vec{\Delta}|} \sinh ^{2} \frac{\omega}{2}\right)\right] \frac{k_{z}^{\prime}}{k^{\prime}} t_{0}(k) t_{1}\left(k^{\prime}\right) \\
& \left.+\left[C+\cosh \frac{\omega}{2} \sinh \frac{\omega}{2} \frac{\Delta_{x} \widetilde{\Delta}_{x}}{|\vec{\Delta}| k_{z}^{\prime}}+\frac{\Delta_{x} \widetilde{\Delta}_{x} \Delta_{z}}{t k_{z}^{\prime}}\right] \frac{k_{z} k_{z}^{\prime}}{k k^{\prime}} t_{1}(k) t_{1}\left(k^{\prime}\right)\right\} \\
& \times\left\langle p^{\prime} \lambda^{\prime}\left|b_{+}^{q \dagger} b_{-}^{q}\right| p \lambda\right\rangle,
\end{aligned}
$$

where $C=\cosh ^{2}(\omega / 2)+\left(\Delta_{z}^{2} / t\right) \sinh ^{2}(\omega / 2), k^{\prime} \equiv\left|\vec{k}^{\prime}\right|, \vec{k}^{\prime}=\vec{k}+\overrightarrow{\widetilde{\Delta}}$ and the effective momentum transfer is $\overrightarrow{\widetilde{\Delta}}=\eta \vec{\Delta} / \cosh \omega$. For simplicity, it has been chosen $\vec{\Delta}=\left(\Delta_{x}, 0, \Delta_{z}\right)$. The explicit forms of the spectator term $Z(t)$, of the functions $t_{0}(k)$ and $t_{1}(k)$, of the normalization $N$ and of $k_{z}$ are given in [13]. Besides, in Eq (4), $\cosh \omega=\bar{m} / m, \sinh \omega=|\vec{\Delta}| /(2 m), R$ is the bag radius, related to the quark energy $\epsilon_{0}=\omega_{0} / R$, being $\omega_{0}=2.04$ the lowest frequency solution of the bag eigenequation, given in turn by the relation: $R m=4 \omega_{0}[12,15]$. If $S U(6)$ symmetry is assumed, as it has been done in Ref. [15-17] for bag model calculations of 
parton distributions, or in Ref. [13] for bag model calculations of helicity conserving GPDs, the matrix elements $\left\langle p^{\prime} \lambda^{\prime}\left|b_{+}^{q \dagger} b_{-}^{q}\right| p \lambda\right\rangle$, appearing in Eq. (4), reduce to:

$$
\left\langle p^{\prime} \lambda^{\prime}\left|b_{+}^{q \dagger} b_{-}^{q}\right| p \lambda\right\rangle=\delta_{\lambda^{\prime}+, \lambda-}
$$

It turns out therefore that within the MIT bag model in the lowest energy state in an SU(6) spin-flavour scenario, among the helicity flip amplitudes Eq. (2), $A_{++,--}^{q}$ is the only non vanishing one. This is understood in terms of angular momentum conservation: in order to flip the helicity of the quark keeping fixed the one of the target, one has to assume target orbital angular momentum excitation, impossible in a pure $\mathrm{SU}(6)$ scenario. As a consequence, in the present scheme, from Eq. (2) one gets that the generalized transversity distribution

$$
H_{T}^{q}\left(x, \xi, \Delta^{2}\right)=\frac{1}{\sqrt{1-\xi^{2}}} A_{++,--}^{q}\left(x, \xi, \Delta^{2}\right)
$$

is the only non vanishing GPD with helicity flip.

Numerical results for $H_{T}^{q}\left(x, \xi, \Delta^{2}\right)$, Eq. (6), evaluated using Eq. (4) with the SU(6) condition Eq. (5), are shown in Figs 1 to 3. The results have to be ascribed to the low factorization scale, $\mu_{0}$, corresponding to the model, assumed to be $\mu_{0}=0.4 \mathrm{GeV}$ as in Ref. [13], although it is not possible to fix it from first principles. In Fig. 1, the forward limit of Eq. (6), $H_{T}^{q}\left(x, \xi=0, \Delta^{2}=0\right)$, is shown. As expected, it coincides with the result presented, for the transversity distribution $h_{1}^{q}(x)$, in Ref. [16]. In Figs. 2 and 3, the full $x$ and $\xi$ dependences predicted by Eq. (6) are shown for $\Delta^{2}=-0.5 \mathrm{GeV}^{2}$ and $\Delta^{2}=-1 . \mathrm{GeV}^{2}$, respectively. The value of the parameter $\eta$, fixing the effective momentum transfer, has been taken to be 0.55, as done in Ref. [13] for presenting the results. The SU(6) $u$ flavour distribution would be obtained by multiplying these results by $4 / 3$; the $d$ one by multiplying them by $-1 / 3$. The main features of the results are similar to those obtained for the helicity conserving sector [13]: a) a strong $\Delta^{2}$ dependence mainly governed by the ff; b) a weak $\xi$ dependence, although a mild shift of the peak toward larger $x$ can be observed when $\xi$ increases; c) a little contribution in the ERBL region $(-\xi \leq x \leq \xi)$. Some of these features 
may be artifacts of the model under scrutiny. They could be due to the approximations used. Indeed, in the parton helicity conserving sector, some model studies brought to rather different conclusions, in particular concerning the slow $\xi$ dependence of the results together with a $\Delta^{2}$ dependence mainly governed by the ff (see [3] for a summary of results). For an easy discussion I summarize the approximations hidden in the present approach: i) quarks are in the lowest energy state; ii) the role of antiquarks is disregarded; iii) the dependence of the results on the choice of the reference frame is supposed to be weak; iv) a possible effect of the bag boundary has been neglected; v) momentum conservation is only partially restored by a prescription motivated in Ref. $[20,13]$; vi) the spin-flavor structure has been taken to be $S U(6)$. The model can be enriched in different aspects removing part of the approximations i)-vi), which could lead to different $\xi$ and $\Delta^{2}$ behaviours. Finite distributions $\tilde{H}_{T}^{q}, E_{T}^{q}, \tilde{E}_{T}^{q}$ can be obtained by relaxing the assumption i) and/or the assumption vi); a stronger $\xi$ dependence could be obtained by a more transparent prescription for restoring momentum conservation (approximation v). The present analysis has been motivated in part by the necessity of calculating cross-sections for the process studied in Ref. [8], which could give access to $H_{T}^{q}$. In that case, the main contribution comes from the ERBL region. It will be therefore very interesting to extend the study to antiquark degrees of freedom, relaxing the approximation ii). Besides, to predict realistic cross sections, one has to evolve these low-factorization scale results to experimental scales, according to pQCD. This procedure will produce an enhancement of the distribution in the ERBL region. The outcome of this analysis is compared now with a recent lattice calculation [9]. The first moment of $H_{T}^{q}$ is the "tensor form factor"

$$
A_{T 10}^{q}\left(\Delta^{2}\right)=\int_{-1}^{1} d x H_{T}^{q}\left(x, \xi, \Delta^{2}\right)
$$

yielding at $\Delta^{2}=0$ the quark tensor charge. Lattice data for the tensor ff have been recently reported in Ref. [9], where a dipole fit to them has also been proposed. Having no experimental data on this quantity at disposal, in Fig. 4 I compare the isovector $u-d$ tensor ff Eq. (7), obtained by integrating Eq. (6), with the dipole fit of lattice data provided 
in Ref. [9]. It is seen that the results obtained with the choices $\eta=0.35$ and $\eta=0.55$ lie below the points corresponding to the fit. In Ref. [13], the comparison of the MIT bag model calculation for the electromagnetic ff with experimental data had given a different outcome. In that case, at large $-\Delta^{2}$, data were underestimated by the calculation with $\eta=0.55$ and overestimated by taking $\eta=0.35$. In the figure, the calculated $\mathrm{ff}$ has been divided by 1.35, the value of the isovector tensor charge predicted by the MIT bag model with SU(6) symmetry. For this quantity, the same lattice calculation yields the value 1.09. Recently, positivity bounds on helicity flip GPDs have been derived in Ref. [10]. The strongest bound on $H_{T}^{q}$, Eq. (5.1) of Ref. [10], has been found to be fulfilled by the MIT bag with SU(6) symmetry, in any kinematical region.

In summary, a first calculation of quark helicity flip GPDs has been presented. The analysis is motivated in part by the necessity of estimating cross sections for a physical processes which has been proposed to access generalized transversity. As it has been done in the past to obtain first estimates of different parton distributions, the MIT bag model has been chosen, adopting SU(6) symmetry. As expected, in SU(6) only the generalized transversity distribution is non vanishing; the forward limit is recovered and the main features of the full result, at the low factorization scale of the model, are a weak $\xi$ dependence and a little contribution in the ERBL region. The output of the calculation underestimates lattice data and fulfills recently proposed positivity bounds. This work represents a first step for a full modelling of parton helicity flip GPDs in the quark sector, relevant for phenomenological studies. More realistic estimates will be obtained by relaxing the $\mathrm{SU}(6)$ assumption, taking into account antiquark degrees of freedom, implementing a better prescription for restoring translational invariance, evolving the model results to experimental scales. This will permit to obtain more reliable results in the ERBL region.

I am grateful to B. Pire, for suggesting me to start this investigation and for useful comments. Enlightening discussions with V. Vento are gratefully acknowledged. This work is supported in part by the Italian MIUR through the PRIN "Theoretical Studies of the 
Nucleus and the Many Body Systems". 


\section{REFERENCES}

[1] V. Barone, A. Drago, P.G. Ratcliffe, Phys. Rept. 359, 1, (2002); V. Barone, P.G. Ratcliffe, "Transverse spin physics", River Edge, USA, World Scientific (2003).

[2] D. Müller, D. Robaschik, B. Geyer, F.M. Dittes, and J. Hořejši, Fortsch. Phys. 42, 101 (1994); A. Radyushkin, Phys. Lett. B 385, 333 (1996); X. Ji, Phys. Rev. Lett. 78, 610 (1997).

[3] K. Goeke, M.V. Polyakov, and M. Vanderhaeghen, Prog. Part. Nucl. Phys. 47, 401 (2001); M. Diehl, Phys. Rept. 388, 41 (2003); X. Ji, Ann. Rev. Nucl. Part. Sci.54, 413 (2004); A.V. Belitsky and A.V. Radyushkin, Phys. Rept. 418 (2005).

[4] P. Hoodbhoy and X. Ji, Phys. Rev. D58, 054006 (1998).

[5] M. Diehl, Eur. Phys. J. C 19, 485 (2001).

[6] A.V. Belitsky, D. Müller, and A. Kirchner, Nucl. Phys. B629, 323 (2002).

[7] M. Diehl, T. Gousset, and B. Pire, Phys. Rev. D 59, 034023 (1999); J.C. Collins and M. Diehl, Phys. Rev. D 61, 114015 (2000).

[8] D.Yu. Ivanov, B. Pire, L. Szymanowski, and O.V. Teryaev, Phys. Lett. B550, 65-76 (2002); Phys. Part. Nucl. 35, 67 (2004).

[9] M. Göckeler at al. (QCDSF Collaboration), Nucl. Phys. A755, 537 (2005); Phys. Lett. B627, 113 (2005).

[10] M. Kirch, P.V. Pobylitsa, and K. Goeke, Phys. Rev. D72, 054019 (2005).

[11] M. Diehl and Ph. Hägler, hep-ph/0504175; M. Burkardt, hep-ph/0505189.

[12] A. Chodos, R. L. Jaffe, K. Johnson, C. B. Thorn and V. Weisskopf, Phys. Rev. D 9, 3471 (1974).

[13] X. Ji, W. Melnitchouk, and X. Song, Phys. Rev. D 56, 5511 (1997). 
[14] R.L. Jaffe, Phys. Rev. D 11, 1953 (1975).

[15] R.L. Jaffe and X. Ji, Phys. Rev. D 43, 724 (1991).

[16] R.L. Jaffe and X. Ji, Phys. Rev. Lett. 67, 552 (1991); Nucl. Phys. B 375, 527 (1992).

[17] M. Stratmann, Z. Phys. C 60, 763 (1993); S. Scopetta and V. Vento, Phys. Lett. B 424, $25(1998)$.

[18] S. Scopetta and V. Vento, Phys. Lett. B 460, 8 (1999), Erratum-ibid. B 474, 235 (2000).

[19] I.V. Anikin, D. Binosi, R. Medrano, S. Noguera, and V. Vento, Eur. Phys. J. A14, 95 (2002).

[20] X. Song and J. S. McCarthy, Phys. Rev. C 46, 1077 (1992); M. Betz and R. Goldflam, Phys. Rev. D 28, 2848 (1983). 


\section{FIGURE CAPTIONS}

Fig. 1: The GPD $H_{T}^{q}$, Eq. (6), in the forward limit $\xi=0, \Delta^{2}=0$, giving the transversity distribution $h_{1}^{q}(x)$.

Fig. 2: The $x$ and $\xi$ dependences of the helicity flip GPD $H_{T}^{q}$, Eq. (6), for $\Delta^{2}=-0.5$ $\mathrm{GeV}^{2}$.

Fig. 3: As in Fig. 2, but for $\Delta^{2}=-1$. GeV ${ }^{2}$.

Fig. 4: The isovector $(u-d)$ tensor form factor, Eq. (7), evaluated for $\eta=0.35$ (full) and $\eta=0.55$ (dashed), divided by the isovector tensor charge predicted by the MIT bag model with $\mathrm{SU}(6)$ symmetry, compared with the dipole fit to the lattice prediction given in Ref [9] (dots). 


\section{FIGURES}

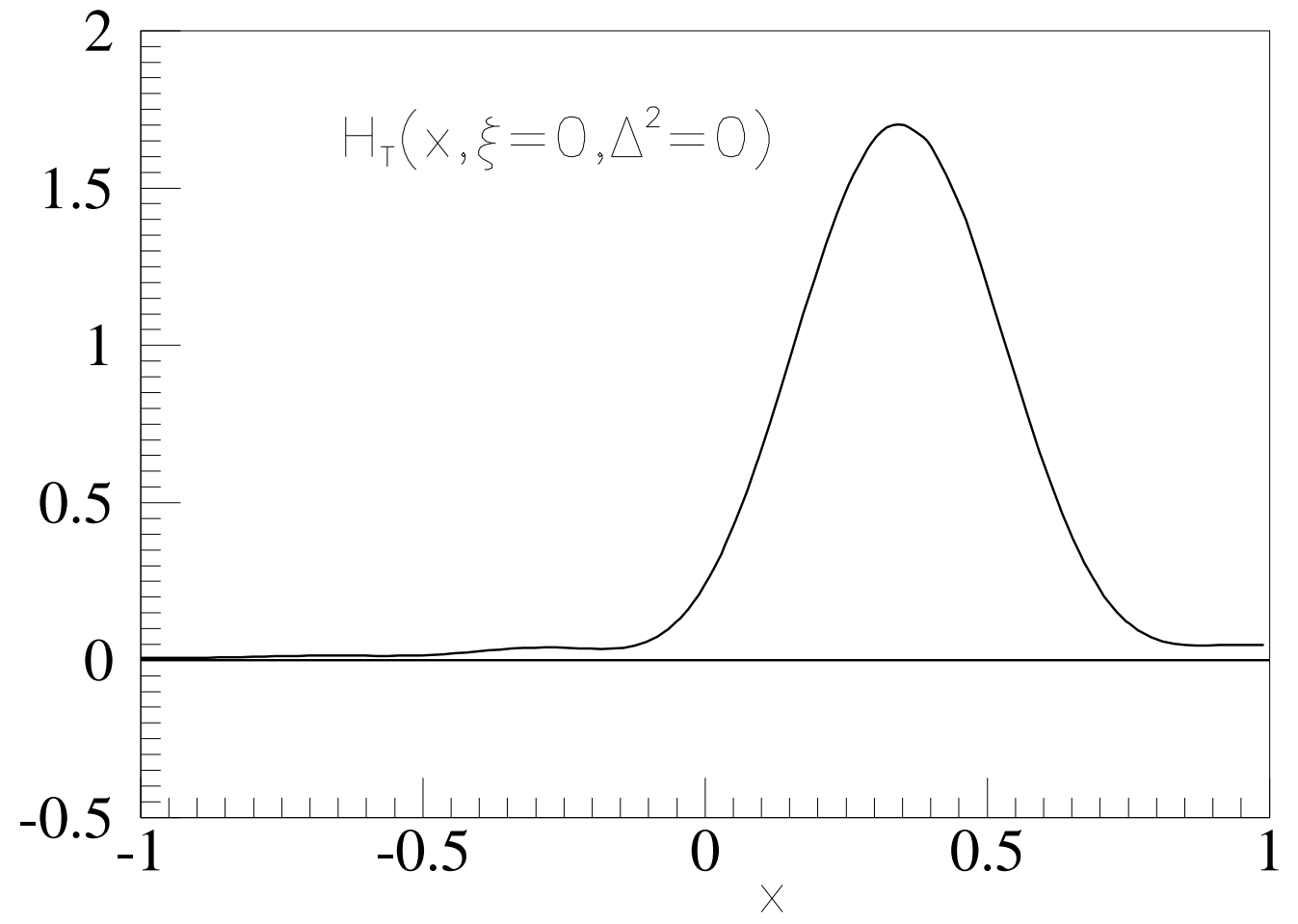

FIG. 1. 
FIG. 2.

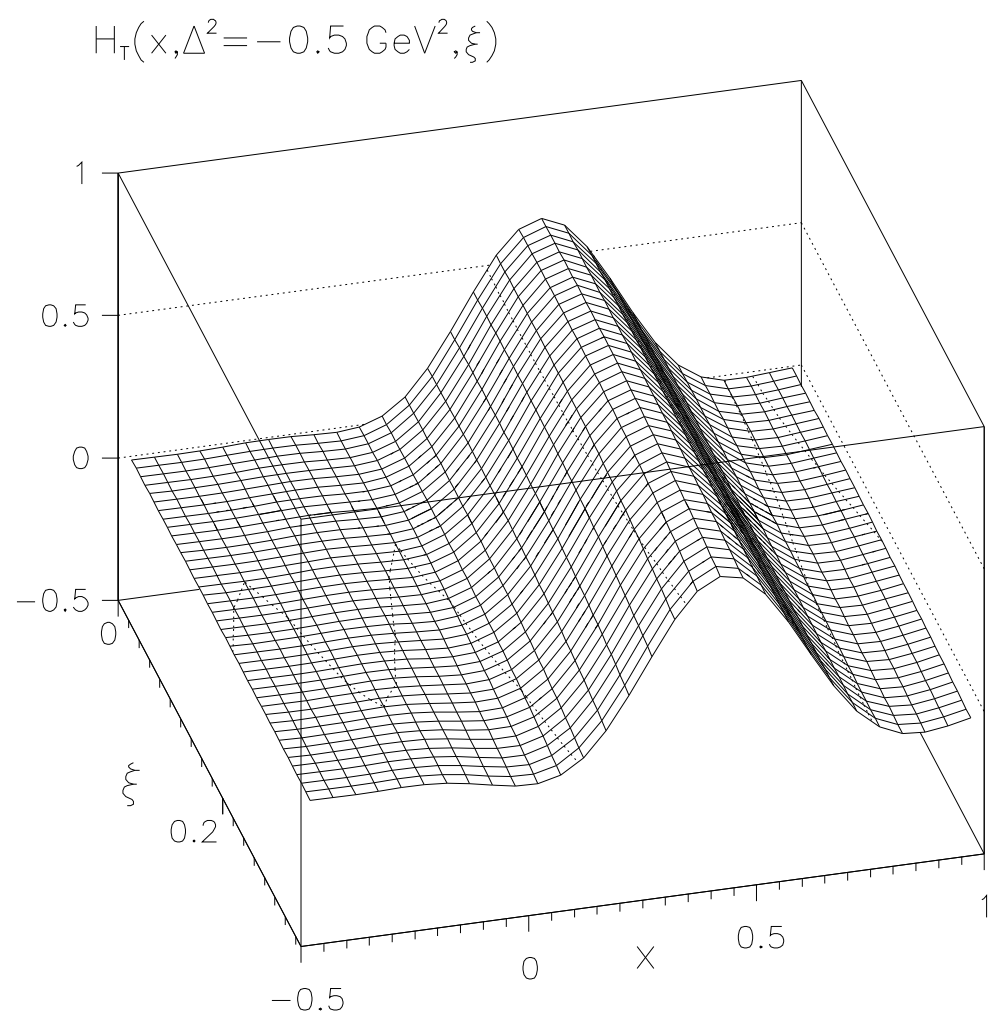




\section{FIG. 3.}

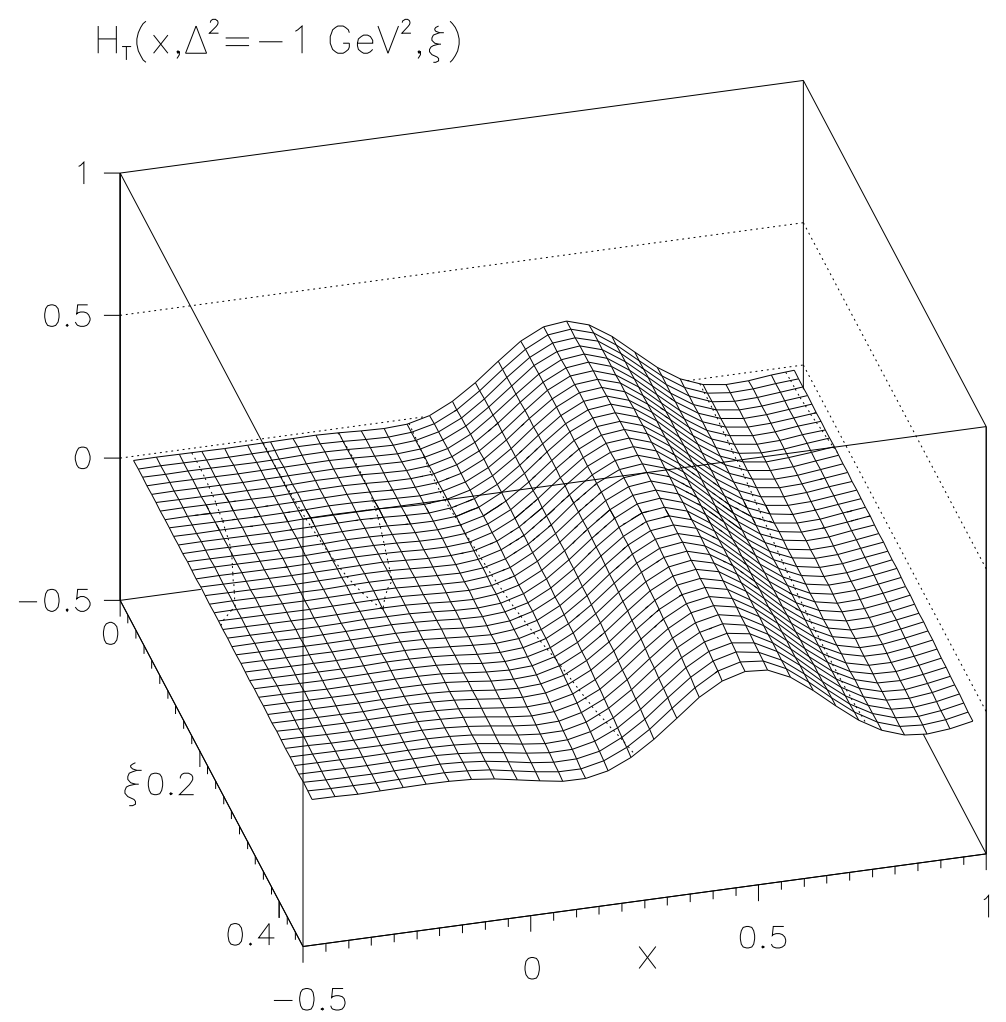


FIG. 4.

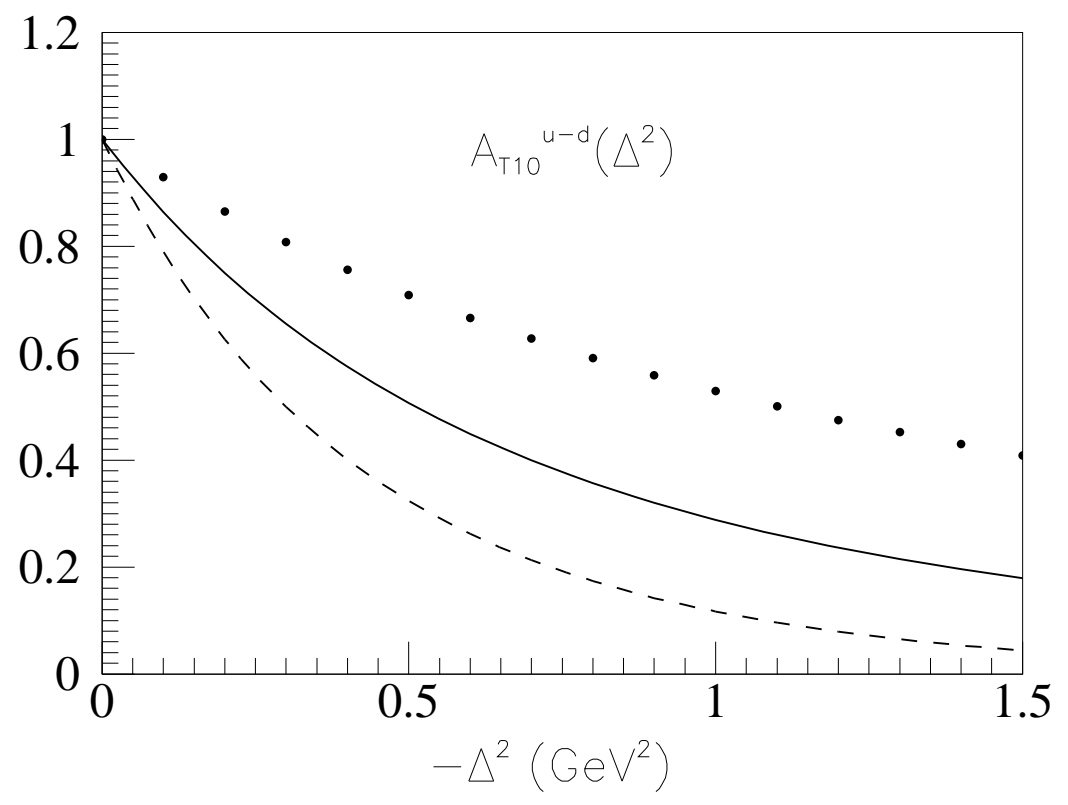

